\title{
Preface
}

\section{Psychiatric Aspects of Critical Care Medicine: Update}

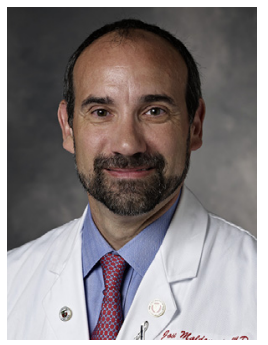

José R. Maldonado, MD, FAPM, FACFE

Editor

Critical care units are fast-paced, stressful environments. They are places where patients are taken to be put back together, but often it is also where they suffer and die. It is likewise a challenging place for medical personnel, carrying a high degree of professional burnout and medical personnel posttraumatic stress disorder (PTSD). This issue represents an ambitious effort to bring together the information that critical care personnel (eg, intensivists, anesthesiologists, surgeons nurses, and medical psychiatrists) need to understand the complex and diverse neuropsychiatric and ethical problems they and their patients face together, every day in the intensive care unit (ICU).

Drs DeGuzman and Ament (Stanford) discuss the epidemiology and neurobiology of traumatic brain injury (TBI), with a special focus on the neurobehavioral sequelae of head trauma, including details regarding TBI's phases of recovery, the evaluation of the resultant neurocognitive sequelae, and a discussion of a rational approach to the management of the behavioral and psychiatric symptoms following trauma.

As the US population ages, clinicians have to deal with an aging brain. Drs Oldham, Piddoubny, Peterson and Lee (Johns Hopkins) provide us with an excellent review of the presentation and detection of preexisting cognitive impairment in the critical care setting and provide the foundation for the management of the behavioral and psychiatric symptoms of cognitive impairment in the clinical setting.

Delirium or acute brain failure is the single most common neurobehavioral disorder experienced by medically ill patients, and no place has a higher incidence than the critical care unit. The occurrence of delirium affects patient's morbidity and mortality, affects the safety of patients and providers alike, and has profound implications regarding long-term outcome. Dr Maldonado (Stanford) conducts a comprehensive review of the epidemiology, etiologic factors, characteristics, and methods of diagnosis and summarizes the evidenced-based data on methods of treating and preventing delirium in the critical care units. 
Drs Rasimas and Sinclair ( $U$ of Minnesota) take us through a thorough description of the most common toxidromes likely to be faced by critically ill individuals, either because some form of poisoning got the patient to the ICU or because of the toxic effects of medical treatment. They do a masterful job discussing the interaction between toxicity, neuronal function, and mental status alteration, while guiding us through a maze of potentially deadly consequences of drug toxicity and how to help our patients survive them.

Drs Tetrault and Donroe (Yale) review the epidemiology of substance abuse and how it may contribute and/or complicate the treatment of the patient's underlying medical condition. Their article discusses both iatrogenic substances of abuse, in which doctors are partly complicit, and street drugs, such as cocaine, amphetamines, MDMA, synthetic cannabinoids, and bath salts, and their complications and provide clinical recommendations regarding the treatment of related conditions.

Dr Maldonado (Stanford) discusses the second most common substance use problem in the United States, alcohol use disorder, with an emphasis on the various withdrawal syndromes that may adversely affect the delivery of care in the critical care setting. Many clinicians have faced the problem of an agitated, withdrawing patient who is either requesting "more valium" or experiencing respiratory depression due to the central nervous system depressant effect of benzodiazepines. He discusses tools for the prediction of patients at risk for complicated alcohol withdrawal. $\mathrm{He}$ then provides a roadmap for the use of a Benzodiazepine-sparing protocol, designed to tackle the neurobiology of alcohol withdrawal, while avoiding the complications of conventional treatment approaches.

Dr Sher (Stanford) discusses the highly emotionally charged topic of lung disease and its relationship to psychopathology. Psychiatric conditions are prevalent among patients suffering from chronic lung disease, with rates of depression and anxiety significantly higher than the general population. She then provides a framework for the management of these psychiatric syndromes in the context of the highly emotionally charged setting of the critical care unit.

Similarly, Dr Shapiro (Columbia U) summarizes the specific psychiatric challenges experienced by patients with heart disease in acute, intensive, and critical care. $\mathrm{He}$ focuses on the strong emotional reactions experienced by patients admitted to cardiac critical care, including patient's psychological reactions to the various assistive technologies, such as mechanical ventilation, ventricular-assist devices, and defibrillation, ending with a discussion of the various psychiatric scenarios clinicians are likely to encounter in this setting.

Dr Garcia (Stanford) addresses the emotional and difficult topic of suicide, including predisposing factors leading to it and its neurobiology, and discusses the complexities of the assessment and management of suicide and its aftermath. Different from many other topics in this issue, this is one possible exception when the patient involuntarily ends up in the ICU, because he survived an attempt at ending his life.

The various conditions leading to an acute care admission and the environment and procedures performed in a critical care unit may render patients, their family members, and the staff liable to suffer any number of anxiety symptoms. Drs Bienvenu and Gerstenblith (Johns Hopkins) focus on one form of psychiatric morbidity in critical illness survivors, PTSD, which affects nearly $20 \%$ of critical illness survivors. They discuss the risk factors, treatment modalities, and what ICU personnel can do to prevent this disorder.

The ICU team plays an integral part in the medical journeys of transplant recipients. The critical care unit serves as the threshold between the failed organ and their new life. The ICU is the place where they wait for a new organ or die waiting for 
one. Drs Sher (Stanford) and Zimbrean (Yale) discuss the most up-to-date information on the psychosocial aspects of transplant patients in the critical care setting, including pretransplant evaluation, psychological considerations of assist devices in the ICU, peritransplantation neuropsychiatric syndromes, and relevant aspects of their psychopharmacology, including potential drug-drug interactions.

Drs Munjal and Ferrando (New York Medical College) and Dr Freyberg (Columbia) provide a detailed review of a select number of infectious agents with predilection to the central nervous system, which may lead to or complicate the assessment and management of ICU patients. In particular, they focus on HIV/AIDS, herpes encephalitis, pediatric acute-onset neuropsychiatric syndrome or childhood acute neuropsychiatric symptoms (formerly known as PANDAS), neurocysticercosis, neurosyphilis, Lyme disease, and Creutzfeldt-Jakob disease. In the process, they discuss their neuropsychiatric manifestations, diagnosis, and treatment.

Psychotropic agents are commonly used in the critical care setting, often serving as an indispensable adjunct to manage many neuropsychiatric and behavioral conditions, which present as a complication of medical treatment or represent part of the patient's substrate. In any case, psychoactive agents can present with any number of life-threatening situations, including serotonin syndrome, neuroleptic malignant syndrome and other forms of extrapyramidal syndromes, cardiac dysfunction, endocrine disturbances, discontinuation syndromes, and others. Drs Lahijani (Stanford) and Harris (Rush) discuss the most likely presentation, diagnostic techniques, and state-of-the-art management tips for each of these conditions.

We finish with a discussion of palliative and end-of-life care. This is a core component of critical care medicine and addresses the multiple domains of suffering that patients, families, and medical personnel experience. Dr Harman (Stanford) discusses the characteristically comprehensive approach of the palliative medicine specialist, with their unique role as consultants and their emphasis on patient/family-centered care. Often addressing and bridging complex ethical and end-of-life issues, her discussion provides a great conclusion to our attempt to integrate psychiatry and hightech medicine in one comprehensive issue.

I am immensely honored for the opportunity to work with such a wonderful group of dedicated professionals and humbled by the passion, dedication, skill, and wisdom each of my colleagues brings with their contribution to the field of medicine and patient care.

José R. Maldonado, MD, FAPM, FACFE Psychosomatic Medicine Service Emergency Psychiatry Service Stanford University School of Medicine 401 Quarry Road, Office \#2317 Stanford, CA 94305, USA 